\title{
Non-locality bursts into life
}

The past few years have seen a resurgence of interest in the foundations of quantum mechanics, not least because there has been a succession of generations.

THAT we seem to be in for another bout of argument about the interpretation of quantum mechanics is plain, to judge from what has been appearing in the physics journals, but why now, more than half a century after Nils Bohr is believed to have settled everything? There are several explanations. Bohr did not quieten all doubters, among whom Einstein was one (most often represented by the gedanken experiment due to Einstein, Podolski and Rosen, or EPR for short). Nor was de Broglie ever reconciled to the Copenhagen view; there remains an active school of researchers devoted to his view.

Much more recently, it has been recognized that Bohr plucked from thin air the assumption that the square of the (generally complex) amplititude of a solution to the wave equation represents the probability distribution of the particle or other entity concerned, however successful interpretations based on it may be. The recent resurgence of interest thus at least partly springs from the drive to make such leaps in the dark intelligible.

This seems the spirit in which people have been testing the old principle that quantities or "observables" represented by mathematical operators that do not commute with each other cannot be measured simultaneously. From that it follows that it is possible to know only two components of the angular momentum of a particle, perhaps the components in two distinct directions, but more usually the component in one direction and the total angular momentum (which is a number).

The now-standard drill for doing that stems from the fact that any relationship between operators representing physical observables must be mirrored in an identical relationship between values assigned (as if by measurement) to the same quantities, when it becomes a matter (not always simple) of showing that the allowable numbers cannot be consistently assigned to all of a set of physical variables.

But a more immediate stimulus of interest among experimentalists in the interpretation of quantum mechanics has been the publication (itself now more than a quarter of a century ago) of the late J. S. Bell's now-famous inequality - a numerical relation between the probability that physically distant events will be correlated with each other.

Non-locality is the new game, as the EPR experiment dictates. Einstein was not the only one offended that the several components of a single well-defined quantum state may be physically separated yet still bound together by the overall properties of the quantum state.

A simple example is that of two electrons more or less at rest in a singlet state, which means that their net spin momentum is zero, or that (if the term has meaning) the two electron spins are oppositely directed. So what happens if the two electrons are flung diametrically apart by some agency or another? Although the random direction in which the two spins cancel is still undetermined, the cancellation persists indefinitely. And if, at some stage, the projection of the angular momentum of one operator in some direction is measured, one can confidently infer the exact opposite for the corresponding component of the other.

Einstein's protest was that this state of affairs cannot make sense. By what physical means can one electron "know" how to satisfy the overall requirements of the quantum state once a decision has been made to measure the angular momentum of its partner? Whence (in part) the doctrine of hidden variables - the assertion that each of the two separating electrons must carry the information determining its behaviour, consistently with that of its partner, when it encounters a piece of equipment that requires the direction of its spin to be declared.

Attempts to investigate this behaviour experimentally are bound, of course, to be frustrating. With the same singlet state of the electron pair, one can think (most of this is still gedanken stuff) of using a strong magnetic field to allow electrons to pass if they are polarized in the direction of the field, but which rejects them if they are oppositely polarized. One can be sure that both members of a singlet electron pair will each pass through a magnet in its path only if the fields are in opposite direction.

Most simply, the outcome of such an experiment can be represented by the product of two numbers, one for each magnet, in which one scores +1 for passage and -1 for failure. Allowing for arbitrary directions of the magnetic field, say $\mathbf{a}$ and $\mathbf{b}$, the general quantum result is that the expectation value of the product of these two numbers (or the average over many individual measurements) is simply the negative of the scalar product a.b.

Bell's tongue-in-cheek inequalities arise from supposing that the two electrons leave the tightly bound singlet state with a nearly perfect memory of where they have come from, but that they then evolve independently. The numerical inequality is derived by a comparison of the two states, represented by the directions a and $\mathbf{b}$, with a third, say $\mathbf{c}$. The correlation (or anticorrelation) is less strong.

Daniel I. Fivel from the University of Maryland at College Park has now brought many of these arguments together in what seems to be a splendid piece of rationalization by generalization (Phys. Rev. Lett. 67, 286; 1991). All these discussions seem to hang on correlating what happens at one measuring device with what happens at another. So the expectation value of a result with orientation $\mathbf{a}$ at one magnet and orientation $\mathbf{b}$ at the other is a number that might be thought to connect together all pairs of points on the surface of a sphere. (It turns out to be simplest to get rid of negative numbers by adding +1 to the expectation value and dividing the result by two.)

The function that results has all the properties of a metric (for measuring distances on the surface of a sphere) and Bell's inequality turns out to be just the triangle inequality (that the sum of two sides must be greater than the third). But Fivel is also able to show that the assumption that only local issues matter, perhaps helped along by hidden variables, lead to the construction of a metric incompatible with the first. It looks as if the outlook for the EPR way of working may be doomed.

But that will not prevent further experiment. Indeed the same issue of the same journal contains an argument (by J. D. Franson of Johns Hopkins University) that non-locality can be demonstrated by intereference between the photons of two light beams manipulated so that they reach the detectors at slightly different times, and an intriguing experiment with a pair of nonlinear crystals (lithium iodate) by a group from the University of Rochester in which unexplained non-locality is destroyed by preventing the stimulation of one crystal by light from the other. For piquancy, there is also a protest by P.R. Holland and J.P.Vigier from the University of Paris at what they consider to be a misrepresentation of de Broglie.

There is ample evidence that the preoccupation will not go away. This does not mean that people are worrying about the foundations, but that they wish to understand them better. A new generation has arrived.

John Maddox 